IGCS19-0409

\section{ESR1 HOTSPOT MUTATIONS IN ENDOMETRIAL STROMAL SACROMAS MAY CONFER HORMONAL RESISTANCE}

${ }^{1} \mathrm{~K}$ Dessources, ${ }^{2} \mathrm{~A}$ Da Cruz Paula*, ${ }^{2} \mathrm{~S}$ Lee, ${ }^{1} \mathrm{Y}$ Zou, ${ }^{2} \mathrm{R}$ Soslow A, ${ }^{3} \mathrm{M}$ Hensley L, ${ }^{2} \mathrm{~B}$ Weigelt, ${ }^{2} S$ Chiang. ${ }^{1}$ Memorial Sloan Kettering Cancer Center, Surgery, New York, USA; ${ }^{2}$ Memorial Sloan Kettering Cancer Center, Pathology, New York, USA; ${ }^{3}$ Memorial Sloan Kettering Cancer Center, Medicine, New York, USA

\subsection{6/ijgc-2019-IGCS.116}

Objectives Rare low-grade endometrial stromal sarcomas (LGESS) may show high-grade morphology in primary or recurrent tumors. These lesions are classified as high-grade endometrial stromal sarcomas (HGESS), which in general are more aggressive and have higher rates of resistance to endocrine therapy than LGESS. The pathogenesis of hormonal resistance in these tumors has yet to be defined. Here we describe two endocrine-resistant HGESS with ESR1hotspot mutations.

Methods For case 1, DNA from the primary estrogen receptor (ER)-positive LGESS and two ER-positive HGESS recurrences, and for case 2, DNA from an ER-positive recurrent LGESS and ER-positive HGESS recurrence were subjected to sequencing targeting 468 cancer-related genes. RNA from each case was also evaluated for the presence of gene fusions using ARCHER FusionPlex. Sequencing data were analyzed using state-of-the-art bioinformatics algorithms.

Results Both patients received at least two lines of hormonal suppressive therapy including letrozole and megestrol. Cases 1 and 2 harbored JAZF1-PHF1 and EPC1-PHF1fusions, respectively. The primary LGESS and the two HGESS recurrences of case 1 shared MST1,KDM5Cand ARID1Bmutations; however in the second HGESS recurrence post endocrine treatment, clonal STK40(R128W) and ESR1hotspot (Y537S) mutations were detected. In contrast, both LGESS and HGESS recurrences of case 2 harbored a LATS2mutation and a clonal ESR1Y537S hotspot mutation. In addition, an HRASQ61R hotspot mutation restricted to the recurrent LGESS was identified.

Conclusions Our findings suggest that the ESR1Y537S hotspot mutation in LGESS, either pre-existing or acquired, may be associated with endocrine resistance and/or high-grade transformation in these lesions.

\section{IGCS19-0091}

\section{COOPERATIVE EFFECT OF HUMAN PAPILLOMAVIRUS TYPE 18 E5, E6 E7 ONCOPROTEINS IN PROMOTING CELL PROLIFERATION, MIGRATION, INVASION AND IN MODULATING CELLULAR REDOX STATE}

\footnotetext{
${ }^{1} \mathrm{JP}$ Hochmann Valls*, ${ }^{1} \mathrm{~F}$ Parietti, ${ }^{2} \mathrm{~J}$ Martinez, ${ }^{3} \mathrm{AC}$ Lopez, ${ }^{3} \mathrm{M}$ Carreño, ${ }^{2} \mathrm{C}$ Quijano, ${ }^{4} \mathrm{~L}$ Sichero, ${ }^{3} \mathrm{M}$ Möller, ${ }^{1} \mathrm{~S}$ Mirazo, ${ }^{1} \mathrm{~J}$ Arbiza. ${ }^{1}$ Sección Virología, Facultad de Ciencias, Universidad de la República (UDELAR). Montevideo-Uruguay; ${ }^{2}$ Centro de Investigaciones Biomédicas (CEINBIO and Departamento de Bioquímica, Facultad de Medicina, Universidad de la República (UDELAR). Montevideo-Uruguay; ${ }^{3}$ Laboratorio de Fisicoquímica Biológica, Facultad de Ciencias, Universidad de la República (UDELAR). Montevideo-Uruguay; ${ }^{4}$ Center for Translational Research in Oncology, Instituto do Cancer do Estado de São Paulo, Hospital das Clinicas da Faculdade de Medicina da Universidade de São Paulo, São Paulo, Brazil
}

10.1136/ijgc-2019-IGCS.117
Objectives The main aim of this work is to study how E5, E6 and E7 oncogenes of human papillomaviruses type 18 could cooperate among each other to boost key cancer cell features such as uncontrolled cell proliferation, enhanced migration capacity, invasion, and how this relates with oxidative stress.

Methods We generated three $\mathrm{HaCaT}$ cell lines, that are spontaneously immortalized, expressing the following combination of oncogenes: HaCaT E5-18, HaCaT E6/E7-18 and $\mathrm{HaCaT}$ E5/E6/E7-18 and non-transduced HaCaT cells as a control. Cell proliferation was assessed using a MTT assay. Cellular migration was studied using a wound healing assay by scratching the monolayer in order to generate a "wound". Invasiveness potential of cells was studied using a transwell collagen invasion assay. Intracellular oxidants production in the four cell lines was measured using the fluorescent probe CM-H2DCFDA. Catalase activity was assayed spectrophotometrically, following the decomposition of $10 \mathrm{mM} \mathrm{H}_{2} \mathrm{O}_{2}$ by catalase contained in the samples at $240 \mathrm{~nm}$. Total glutathione in cell lysates was quantified by HPLC, and PRX1 expression levels was assessed by Western Blot.

Results MTT assay showed a statistically significant increment in cell proliferation in $\mathrm{HaCaT}$ E5/E6/E7 cell with respect to the other three cell lines. Similar results were obtained in cell migration assay, and in invasion assays. We measured levels of the three oncoproteins involved in ROS metabolism and observed that E5/E6/E7 diminished catalase activity but augmented significantly the levels of GSH and PRX1.

Conclusions This study demonstrates that cells with E5/E6/E7 together cooperate to augment the malignant transformation of HaCaT cells.

\section{IGCS19-0385}

\section{CDK12 REGULATES GENE EXPRESSION OF DNMT1 AND ERBB3 BY ALTERING TRANSCRIPTION OF MIR-152}

1J Klat*, ${ }^{2}$ M Dzimkova, ${ }^{2} \mathrm{H}$ Paculova, ${ }^{2}$ K Kohoutek. 'University Hospital Ostrava, Ob/Gyn, Ostrava, Czech Republic; ${ }^{2}$ Veterinary Research Institute, Department of Chemistry and Toxicology, Brno, Czech Republic

\subsection{6/ijgc-2019-IGCS.118}

Objectives The DNA-damage-response (DDR) pathway is a cellular mechanism which has evolved to protect cellular integrity by detection and repair of DNA lesions. Cyclindependent kinase 12 (CDK12) maintains genome stability via regulation of transcription of DDR genes, specifically, BRCA1, RAD51. Importantly, down-regulation of the CDK12 caused induction of the 53BP1 and $\gamma \mathrm{H} 2 \mathrm{AX}$ foci and accumulation of cells in the G2-M phase of the cell cycle. Since various microRNA (miRNA) are situated within coding genes, such as DDR, we hypothesize that expression of some of them might be also affected by CDK12 depletion.

Methods A pilot study focused on identification of candidate miRNAs in ovarian cancer cells that might be significantly altered in CDK12 deficient cells. Indeed, downregulation of CDK12 protein level led to aberrant expression of several miRNAs. Among studied miRNAs, the level of miR-152 was significantly elevated. By using 